\title{
Use and utility of a 24-hour Telephone Support Service for 'high risk' patients with COPD
}

\section{*John R Hurst ${ }^{\mathrm{a}, \mathrm{b}}$, Fiona Fitzgerald-Khan ${ }^{\mathrm{b}}$, Jennifer K Quint ${ }^{\mathrm{a}}$, James JP Goldringa, Christine Mikelsons ${ }^{b}$, J Paul Dilworth ${ }^{b}$, Jadwiga A Wedzicha ${ }^{\mathrm{a}, \mathrm{b}}$}

\author{
a Academic Unit of Respiratory Medicine, Royal Free Campus, UCL Medical School, London, UK \\ ${ }^{b}$ Department of Respiratory Medicine, Royal Free Hampstead NHS Trust, London, UK
}

Received 27th October 2009; resubmitted 25th January 2010; revised 8th March 2010; accepted 2nd May 2010; online 22nd June 2010

\begin{abstract}
Background: Hospitalisations are important events in COPD, and exacerbation prevention strategies are not completely effective. Experience with our research cohort suggested that availability of 24-hour telephone advice may reduce hospital admission.

Aim: To examine the use and utility of a 24-hour Telephone Support Service for high-risk NHS COPD patients.

Method: 74 patients with 'high-risk' COPD had therapy optimised, were educated about exacerbations, given home 'emergency' therapy, and had 24-hour access to telephone advice.

Results: Patients had a mean (SD) age of 70.4 (9.1) years and severe disease (mean FEV 1.00 (0.37) litre; 30\% had home oxygen and $46 \%$ lived alone). There were 258 telephone calls in 22,074 follow-up days. $76 \%$ of calls were received between 0800 and 1700 hours. The proportion of possible exacerbation ('appropriate') calls (overall 56\%) was higher at weekends and overnight. Overnight calls (21000800) were rare: to expect one appropriate call per shift would require 2453 patients. A third of appropriate overnight calls could be managed without further emergency assessment. Mean (SD) length of follow-up was 298 (117) days/patient. Patients completing one year of follow-up $(n=52)$ demonstrated a $45 \%$ reduction in admissions and $37 \%$ reduction in bed days. Patient satisfaction was high.

Conclusions: We report data on the use and utility of a 24-hour Telephone Support Service in COPD. The service was associated with a reduction in hospital admission. Call volume was low, thus giving information on the size and cost-effectiveness of such service provision.

(C) 2010 Primary Care Respiratory Society UK. All rights reserved.

JR Hurst et al. Prim Care Resp J 2010; 19(3): 260-265

doi:10.4104/pcrj.2010.00035
\end{abstract}

Keywords COPD, exacerbation, hospitalisation, telephone support, service development

\section{Introduction}

Chronic obstructive pulmonary disease (COPD) is a prevalent and disabling condition that results in significant personal impact to patients and their carers, and financial cost to health services. ${ }^{1}$ More than half of these costs relate to hospital admissions for acute exacerbation. ${ }^{2}$ Reducing exacerbations and hospitalisations are therefore key goals in COPD management. ${ }^{1}$

There is now a range of pharmacological and nonpharmacological interventions that can reduce exacerbations in COPD. ${ }^{3}$ However, even when the best combination of interventions is selected for an individual patient some exacerbations still occur. Early treatment of an exacerbation accelerates recovery, ${ }^{4}$ and therefore easier access to care at the start of an exacerbation may also reduce hospitalisation. However, COPD patients are also prone to multiple comorbidities which may present with symptoms indistinguishable from exacerbations. ${ }^{5}$ The reasons why patients with COPD and acute symptom deterioration present to hospital are therefore complex. Moreover, appropriate information about such patients is rarely available to frontline staff, which also likely contributes to hospital admission. This all suggests that self-management, in partnership with health-care professionals, could reduce exacerbations and hospitalisations. A systematic review ${ }^{6}$ has recently concluded that chronic care models in COPD can indeed reduce

* Corresponding author: Dr John R Hurst, Academic Unit of Respiratory Medicine, UCL Medical School, Royal Free Campus, London, NW3 2PF, UK Tel: +44 (0)207317 7510 Fax: +44 (0)2074726141 E-mail: j.hurst@medsch.ucl.ac.uk 
hospitalisations when they include two or more of four key components termed Self-Management, a Delivery System Design, Decision Support and a Clinical Information System. Our own experience running a COPD research cohort demonstrated that educating patients about exacerbations and providing a 24-hour telephone advice service resulted in relatively few hospital admissions. This intervention is significantly less complex and more practical than many of those employed in previous trials, and we believed that the methodology could be translated to a UK NHS population.

We therefore aimed to establish the use and utility of a 24hour telephone advice (Early Presentation and Intervention in COPD, EPIC) service for COPD patients at high-risk of hospital admission, either through the severity of their underlying disease or because of a previous history of COPD hospitalisation. The model included all four of the key components listed above, focused on the availability of 24-hour telephone access to specialist, personalised advice. Pilot funding was secured from an Innovations scheme in our Trust and, to secure future funding, the service was comprehensively audited. This paper reports that audit, providing the first data relating to the use and utility of a 24-hour telephone advice service for NHS COPD patients. There is an urgent need for such data, given that such advice lines are being established without available knowledge on their usage or the necessary scale to inform cost-effectiveness.

\section{Methods}

\section{Recruitment}

We aimed to approach all patients with 'high risk' COPD attending the Hospital Trust over a six-month period (commencing 10th December 2007). We defined 'high risk' as those patients with a previous hospitalisation for COPD, or very severe disease (GOLD stage IV: FEV $1<30 \%$ predicted and or the presence of respiratory failure). Patients were approached prior to hospital discharge, or in an out-patient setting. Royal Free Hampstead NHS Trust serves a diverse local population, including some of the most deprived and affluent areas of London.

At recruitment, detailed information on COPD severity, exacerbation history, medication and home therapies, comorbidities and social circumstance was collected and entered onto a database. New lung function tests were not specifically performed for the service and historical data was collated. All patients had their therapies optimised in accordance with national guidelines. ${ }^{1}$ Patients were educated about the importance of early therapy at exacerbation, and provided with an emergency course of oral corticosteroid (prednisolone 30mg once daily for 7 days) and antibiotics to have at home. Unless the patient was allergic or intolerant, or there was previous isolation of a resistant organism, the first line antibiotic was amoxicillin 500mg three times daily for 7 days. Patients received written instructions about the service, and were invited to contact us if "you need any advice about your chest, and particularly if you think you may be having a flare-up". There was no separate written action plan. Patients were still free to contact other healthcare providers such as their general practitioner (GP) or community matron. The GP was informed about the patient's participation in the service.

There were no specific exclusion criteria, but in the absence of out-of-hours translation services all patients (or their carer) had to speak English, and have access to a telephone.

\section{Follow-up}

Patients were contacted by telephone two-monthly by the study nurse, and invited to attend an out-patient clinic (as is our usual practice) six-monthly. The aim was for all patients to complete one year of data collection. Data collection stopped on June 10th 2009.

\section{Telephone advice}

In working hours, the telephone was generally manned by the study nurse with back up from the physicians. Out-of-Hours the telephone was generally manned by a physician or, occasionally, by the study nurse supported by a physician. The person manning the phone had immediate access to the patient database. Calls resulting in advice to commence the emergency course of antibiotics and steroids were followed up by further calls as necessary over the following days, and repeat supplies of emergency medication was distributed to the patients.

All calls were logged, including the date and time, duration, nature of the call and clinical outcome. Calls were considered appropriate if they were in response to new symptom deterioration that may have represented exacerbation.

\section{Service outcomes}

This was an audited service development and this report focuses on the use and utility of the 24-hour phone service. We did, however, collect data on the number and length of hospital admissions for patients enrolled in the study for the year of, and year prior to, the intervention. Data were available from the hospital database (both years), patient report (year prior to admission) and study records (year of study). Patient satisfaction with the service was assessed using a five-point questionnaire.

\section{Ethics}

As a service development, ethics approval was not sought. Patients consented verbally to take part in the service.

\section{Results}

\section{Service participants}

Seventy-five patients were invited to take part over the sixmonth period 10th December 2007 to 10th June 2008. One patient admitted with a clinical diagnosis of COPD exacerbation did not have airflow obstruction on subsequent spirometry, leaving 74 subjects in total. All the remaining participants fulfilled the WHO GOLD criteria for COPD, and 
had a clinical diagnosis of COPD (rather than asthma) from a consultant respiratory physician. To assess the proportion of available patients approached, we carefully examined the number of patients admitted or discharged from hospital with a primary diagnosis of COPD exacerbation over the six-month recruitment period; 97 patients fulfilled these criteria, of whom 31 were recruited, and we therefore approached $32 \%$ of the available admissions. The rest of the patients were approached during out-patient clinics and pulmonary rehabilitation classes. The major reasons that patients were not approached included short admissions (particularly over weekends), the presence of a more unstable co-morbidity (for example malignancy receiving palliative care), or an inability to access the service appropriately, notably through cognitive impairment. Patients with stable comorbidities such as cardiac failure and diabetes were included. Two patients declined to accept the service.

The characteristics of the enrolled patients are reported in Table 1, demonstrating that they were a 'high risk' population: 70/74 (95\%) had either GOLD stage IV disease and/or had been previously hospitalised for an exacerbation. All 34 GOLD stage 4 patients had been previously hospitalised. 30\% received long-term oxygen therapy (LTOT) and $46 \%$ lived alone.

\section{Follow-up}

Fifty-two patients (70\%) completed one year of service use, 17 died $(23 \%)$, and five left the service through request or because they moved out of the area (7\%). In total, the 74 patients were followed up on the service for 22,074 days, a mean (SD) of 298 (117) days/patient. The 17 patients who died were older ( 75.3 vs. 68.4 years, $p<0.001$ ) but did not differ from the 52 survivors by sex, lung function variables, oxygen saturation, self-reported exacerbation frequency, body mass index, current or total smoke exposure, or whether they lived alone.

\section{Use of the Telephone Support Service}

Calls were received from, or were regarding, 56 of the 74 patients $(76 \%)$, including 12 of the 17 patients who died (71\%). There were a total of 258 calls, lasting 2,043.5 minutes, at a mean duration of 7.9 minutes/call. This represents a call frequency of 0.01 calls/patient/day (258/22074). There would therefore be one call per day for every 100 patients enrolled on such a service.

Whilst most of the calls ( $n=203,79 \%)$ were made by patients themselves, other people also used the telephone service including carers ( $n=45$ calls, 17\%) and healthcare professionals $(n=10,4 \%$, including community matrons $(n=6), G P s(n=3)$ and paramedics $(n=1))$.

- Characteristics of Patients who did and did not use the phone service

The 56 patients who used the telephone service did not differ from the 18 who did not in age, sex, lung function variables (and therefore disease severity), pulse oximetry, self-reported
Table 1. Characteristics of the $\mathbf{7 4}$ patients enrolled on

the service.

\begin{tabular}{|c|c|c|}
\hline & Mean & SD \\
\hline Age (years) & 70.3 & 9.1 \\
\hline $\mathrm{BMI}\left(\mathrm{kg} / \mathrm{m}^{2}\right)$ & 25.1 & 7.4 \\
\hline Smoking (Pack Years) & 53.9 & 31.2 \\
\hline \multicolumn{3}{|l|}{ Lung Function* } \\
\hline $\mathrm{FEV}_{1}(\mathrm{I})$ & 1.00 & 0.37 \\
\hline $\mathrm{FEV}_{1} \%$ predicted & 44.2 & 16.5 \\
\hline FVC (I) & 2.37 & 0.79 \\
\hline FVC \% predicted & 80.3 & 23.8 \\
\hline \multirow[t]{2}{*}{$\mathrm{FEV}_{1} / \mathrm{FVC}$} & 0.44 & 0.13 \\
\hline & $\mathrm{n}$ & $\%$ \\
\hline Female Sex & 37 & 50 \\
\hline Current Smoker & 21 & 28 \\
\hline GOLD Stage $1 / 2 / 3 / 4$ & $1 / 19 / 19 / 34$ & $1 / 26 / 26 / 46$ \\
\hline LTOT (long term oxygen therapy) & 22 & $30 \%$ \\
\hline \multirow[t]{2}{*}{ Lives Alone } & 34 & $46 \%$ \\
\hline & Median & IQR \\
\hline Self-Reported Exacerbations in previous year & 3 & $1-4$ \\
\hline \multicolumn{3}{|c|}{$\begin{array}{l}\text { * stable results, obtained during the service period in } 27(36 \%) \text {, or a } \\
\text { median (IQR) of } 8(2-24) \text { months prior to enrolment in } 46(64 \%) \text {. Lung } \\
\text { function data was not available in one patient with recurrent pneumothorax } \\
\text { who had extensive emphysema on CT scanning. }\end{array}$} \\
\hline
\end{tabular}

Figure 1. Distribution of calls by time of day.

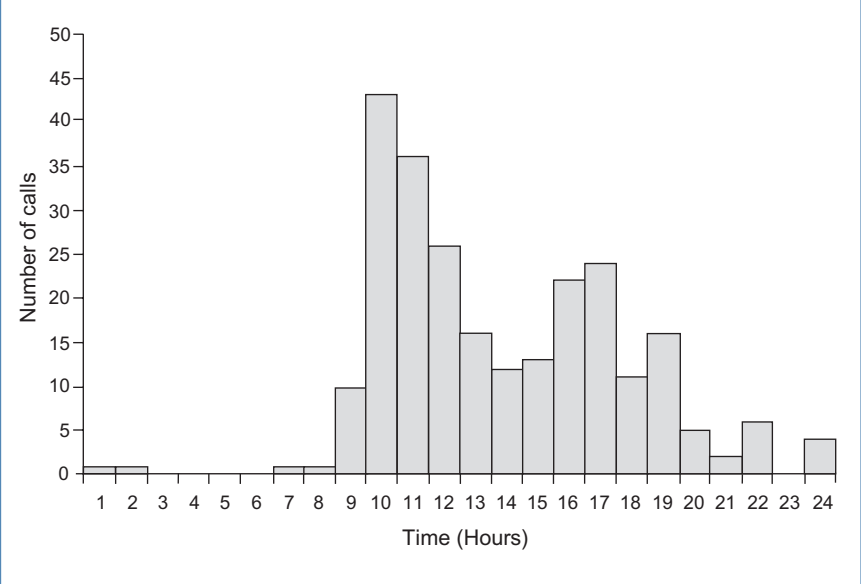

exacerbation frequency, body mass index, current or total smoke exposure, whether they lived alone, or the likelihood of death whilst on the service.

- Appropriateness of calls and variation in calls by time, day and month

The distribution of calls by time of day is illustrated in Figure 1 , demonstrating a bimodal distribution with peaks between 0900-1000 and 1600-1700 hours. The majority of calls (76\%) were received in usual office hours (0800-1700), with much fewer in the evenings, overnight and at weekends. 


Table 2. Distribution and appropriateness of calls by
time and day.
\begin{tabular}{lcccc} 
n (\%) & $\begin{array}{c}\text { Mon-Fri } \\
0800-1700\end{array}$ & $\begin{array}{c}\text { Sat-Sun } \\
0800-1700\end{array}$ & $\begin{array}{c}\text { Any Day } \\
1700-2100\end{array}$ & $\begin{array}{c}\text { Any Day } \\
2100-0800\end{array}$ \\
\hline Appropriate Calls & $104(53 \%)$ & $17(71 \%)$ & $14(52 \%)$ & $9(82 \%)$ \\
\hline Other Calls & $92(47 \%)$ & $7(29 \%)$ & $13(48 \%)$ & $2(18 \%)$ \\
\hline TOTAL & 196 & 24 & 27 & 11 \\
\hline
\end{tabular}

Table 3. Mean number of calls per patient per shift.

\begin{tabular}{lcc} 
Calls/pt/shift $(\mathrm{n}$ for 1$)$ & Appropriate & Other \\
\hline Mon-Fri 0800-1700 & $0.007(152)$ & $0.006(171)$ \\
\hline Any Day 1700-2100 & $0.0006(1,577)$ & $0.0006(1,698)$ \\
\hline Any Day 2100-0800 & $0.0004(2,453)$ & $0.0001(11,037)$ \\
\hline Sat-Sun 0800-1700 & $0.003(371)$ & $0.001(901)$ \\
\hline
\end{tabular}

One hundred and forty-four out of 258 calls (56\%) were considered appropriate (in response to deteriorations in respiratory symptoms), and 114 (44\%) were not. The proportion of calls that were appropriate differed by the time of call (see Table 2), with a higher proportion of appropriate calls on weekend days (71\%) and overnight (82\%) compared to weekday working hours (53\%) or evenings (52\%).

There was no difference in the number of appropriate calls received by day of the week (ANOVA $p=0.330$ ). This suggests that patients were just as likely to report symptom deteriorations via the telephone service on weekend days compared to weekdays. There were significant differences by day in the number of other calls and therefore total calls, $(p=0.001$ and $p=0.002$, respectively), which were significantly less frequent at weekends.

These data also inform on the number of calls per patient per shift, and therefore the size of the population using the service that would generate one call. This is reported in Table 3. A service would require 2,453 patients enrolled to expect one call per overnight shift in response to a deterioration in symptoms.

Because recruitment took place over a six-month rather than one-year period, we did not analyse call frequency by month to inform on seasonal changes.

\section{Utility of the Service}

We examined the number and total duration of hospitalisations for which the primary discharge diagnosis was exacerbation of COPD, in the year of, and year prior to enrolment for the 52 patients who completed one year on the service. The patients who died were not included in this analysis to avoid potentially over-estimating their number of admissions and hospital stay. In the year prior to intervention the 52 patients were admitted 56 times for a total of 461 days. On service, there were 31 admissions lasting 289 days. This represents a 45\% reduction in
Figure 2. Total number (blue bars) and duration (grey bars) of hospitalisations in the year prior to, and year on the EPIC service for the $\mathbf{5 2}$ patients completing one year (both reductions $p=0.002$ ).

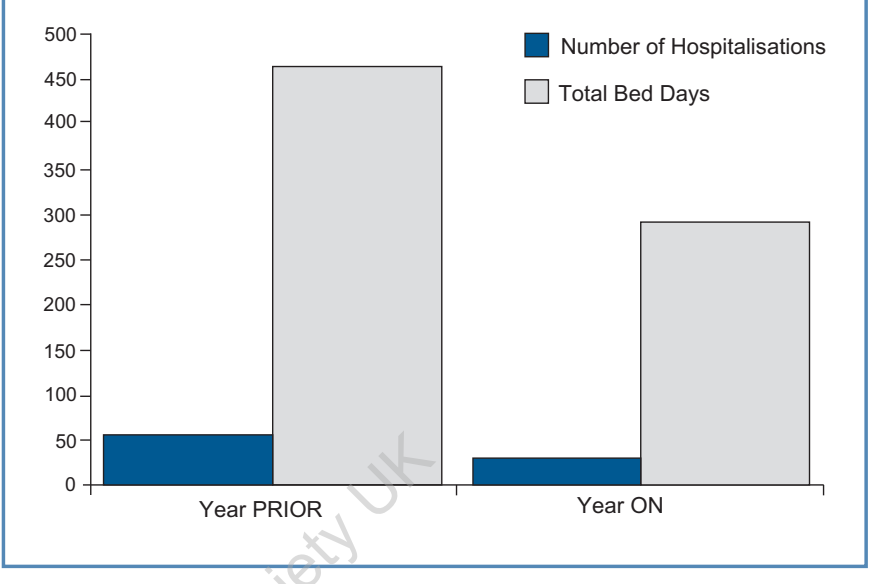

admissions and a $37 \%$ reduction in bed days (both, Wilcoxon $\mathrm{p}=0.002$ ), illustrated as Figure 2. However, for those patients admitted to hospital the mean length of stay increased from 8.2 to 9.3 days, perhaps suggesting that milder exacerbations were being treated in the community.

Of the nine overnight appropriate calls, five resulted in advice to contact emergency medical services (two relating to a patient who had an out-of-hospital cardiac arrest, two resulting in facilitated hospital assessment and admissions, and one resulting in hospital admission for a patient following a fall at home), one was a call from paramedics, two were managed as community exacerbations with their emergency course of therapy, and one was given verbal reassurance only. These latter three episodes all resolved without additional input other than follow-up telephone calls. Therefore, at best, one third of appropriate overnight calls were re-directed away from further emergency assessment.

\section{Satisfaction with the Service}

During the second six months of the service, we aimed to assess patients' views. They were asked, "How would you rate the EPIC service compared to your usual standard of care?". Responses were graded "much better", "a bit better", "the same", "a bit worse" or "much worse". Responses were available for 48 patients (65\%). 46 of the $48(96 \%)$ rated the service as "much better" than standard care. Two patients felt it was the same.

\section{Discussion}

We report the results of an audited service development providing the first data on the use and utility of a 24-hour telephone support service in high-risk UK NHS patients with COPD, and suggesting the potential for this chronic care model to reduce COPD hospitalisations in an NHS setting. However, we suggest that local services at the level of a Primary Care Trust (PCT) may not be cost-effective and therefore such data are 
urgently needed given that many services are currently being proposed or established without an adequate evidence base.

The present study has demonstrated that it may be feasible for such a service to enrol at least one third of all patients admitted to hospital with exacerbation of COPD (it is likely that this could be increased with weekend recruitment) and that additional high-risk patients can be identified from hospital outpatient settings. Three quarters of the patients used the telephone service at some time, generating approximately one call per day per 100 patients on the service. Most of the patients who responded felt that the service was much better than standard care. Most of the calls were from the patients themselves, but carers and health-care professionals also accessed the service. Most calls were during the working day, especially the morning, and there were very few calls overnight. The number of calls in response to symptom changes did not vary by day, but there were more 'administrative' calls on weekdays during office hours and into the evening. A service would require almost 2,500 patients to expect one call overnight in response to deterioration in symptoms, questioning the cost-effectiveness of such provision. Only one in three overnight calls in response to symptom deteriorations can be diverted away from emergency services. However, patients completing one year had fewer hospital admissions and bed days whilst on the service compared to the previous year. We believe that these data support the use of this chronic care model to reduce hospitalisations in 'high-risk' patients with COPD, but suggest that such a service is unlikely to be cost-effective at a local level when provided for 24 hours/day.

The novelty of this report primarily lies in our explicit description of the use of a telephone advice service in COPD, which informs on the necessary size of any future services with regard to cost-effectiveness. There are currently no UK data available on telephone advice services in COPD, despite the establishment of such models. A subsidiary finding was the demonstration of a significant reduction in hospital admissions and length of stay. However, we urge caution in interpreting this result for the following reasons. First, this was not a randomised trial. Second, recruitment of some patients at hospitalisation could bias the previous year results to include more hospitalisation days. Third, not all available patients were recruited, which may have resulted in selection bias.

Why was the service effective? A systematic review ${ }^{6}$ has concluded that chronic care models in COPD reduce hospitalisations when they include two or more of four key components termed Self-Management, a Delivery System, Decision Support and a Clinical Information System. Our model included all four, though with some notable differences from existing studies. Our self-management comprised a single education and recruitment interview (typically taking one hour), written instructions on when to call, and bi-monthly follow-up calls. This is brief when compared, for example, to a previous randomised controlled trial in which the intervention comprised weekly visits for two months, then monthly calls. ${ }^{8}$ Our delivery system was a 24-hour telephone advice line generally manned by a person who knew the patient, who the patient knew, and who was experienced in COPD care. This specialist experience provided intrinsic decision support, and it is important to note that our advice, particularly out-of-hours, was provided by COPD specialists. This may explain why previous nurse-led interventions have not demonstrated clinical benefit. ${ }^{9}$ The nurse in our intervention was supported by a consultant physician with a special interest in COPD, which may limit the generalisability of our findings. However, we do not view specialist knowledge as synonymous with secondary care, and use the term to reflect experience in managing COPD and COPD exacerbations in a range of settings. Finally, our clinical information system comprised a comprehensive database of patient information relating to disease severity, exacerbation history and social circumstance that was available to the healthcare professional at the time of an unplanned call. The availability of this information was key, and would be one way of expanding the service to include healthcare professionals and patients not previously known to each other.

Many alternative models of care have been investigated in COPD, including hospital-at-home schemes focusing on admission avoidance ${ }^{10}$ or early-supported discharge, ${ }^{11,12}$ and selfmanagement strategies. ${ }^{13}$ Our model combined a self- and casemanagement approach, with a focus on admission avoidance. There are many generic problems investigating models of care in COPD, including the small percentage of available patients enrolled, the absence of randomisation, and the introduction of complex interventions. These criticisms can be applied to our EPIC service, and for this reason we have been explicit about such variables throughout this report. It is well described that nonrandomised studies are often more positive than randomised controlled trials. ${ }^{14}$ Nevertheless, our data do inform on service provision. It is not possible to comment on which component of our model was effective, and our intervention included optimisation of medication, education, telephone support, and access to steroids and antibiotics at home.

We believe that a particularly important part of our programme was the detailed patient-specific information available to the healthcare professional manning the telephone, thus enabling appropriate advice to be given. We have previously reported that access to early therapy at exacerbation is associated with faster recovery and reduced risk of hospitalisation ${ }^{4}$ and the present pragmatic clinical data support our previous database analysis. A further important finding was that calls in relation to symptom deteriorations were not less frequent at the weekend, suggesting that the telephone service facilitates access to advice at weekends when it is often more difficult to seek appropriate assistance. 


\section{Summary box}

We present data on the use and utility of a 24-hour Telephone Support Service in COPD, and describe a reduction in hospitalisations associated with a model of chronic care management applied to a high-risk UK NHS COPD population. Practical difficulties in running the service would be the need to access a greater proportion of 'high risk' patients, which would require seven days/week enrolment of patients in secondary care. We suggest that our model should now be assessed in a formal randomised controlled trial. The major lesson for clinical practice would be that such models are liked by patients and may be associated with reduced use of hospital services, but that they may not be cost-effective at the level of individual Primary Care Trusts.

Despite the cost implications, there is some data that patients may specifically value out-of-hours support. ${ }^{15}$ At the time of inception there was no existing information on the use and utility of a 24-hour patient help-line in COPD. However, during the course of our service, a group from Australia have reported their findings. ${ }^{16} 44 \%$ of calls were received in office hours (here defined as Monday-Friday 0900-1700) and 25\% of calls were not related to COPD. In contrast to our study, only one quarter of patients used the service. Patients who called had more severe COPD and were less likely to live alone. Based on patient-reported reduction in calls to emergency services, the authors concluded that such a service was cost-effective (and safe) in reducing hospital attendances. Our patients were likely more severe, and made more use of the telephone service though a greater proportion of the calls were made in office hours. Use of a telephone service will clearly be dependent on the availability of other local services. The systematic review of chronic care models in COPD ${ }^{6}$ reported a pooled $42 \%$ reduction in unscheduled visits, and a $22 \%$ reduction in hospitalisations for trials including more than one of the four components described above. The latter were also associated with a mean reduction in hospital stay of 2.5 days. Our data showed that for those patients admitted to hospital the mean length of stay actually increased from 8.2 to 9.3 days, perhaps suggesting that milder exacerbations were being treated in the community. We specifically targeted high-risk patients, in contrast to other previous studies in which benefit was not demonstrated. ${ }^{17}$

\section{Conclusion}

We report the first data explicitly describing the use and utility of a 24-hour Telephone Support Service in COPD, and describe a reduction in hospitalisations associated with a model of chronic care management applied to a high-risk UK NHS COPD population. The results inform on the applicability, size and cost-effectiveness of such models, and will be of value to those considering, commissioning, establishing and running such services.

\section{Conflict of interest declaration}

None to declare.

\section{References}

1. National Institute for Clinical Excellence (NICE). Chronic obstructive pulmonary disease: national clinical guideline for management of chronic obstructive pulmonary disease in adults in primary and secondary care. Thorax 2004;59(Suppl I).

2. Britton M. The burden of COPD in the UK: results from the confronting COPD survey. Respir Med 2003;97 (Suppl):S71-S79. http://dx.doi.org/10.1016/S09546111(03)80027-6

3. Hurst JR, Wedzicha JA. Management and Prevention of COPD Exacerbations: a state of the art review. BMC Medicine 2009;7:40. http://dx.doi.org/10.1186/17417015-7-40

4. Wilkinson TM, Donaldson GC, Hurst JR, Seemungal TA, Wedzicha JA. Early therapy improves outcomes of exacerbations of chronic obstructive pulmonary disease. Am J Respir Crit Care Med 2004;169:1298-303. http://dx.doi.org/10.1164/rccm. 200310-14430C

5. Hurst JR, Wedzicha JA. What is (and what is not) an exacerbation of COPD: thoughts from the new GOLD guidelines. Thorax 2007;62:198-9. http://dx.doi.org/10.1136/thx.2007.077883

6. Adams SG, Smith PK, Allan PF et al. Systematic Review of the Chronic Care Model in Chronic Obstructive Pulmonary Disease Prevention and Management. Arch Intern Med 2007;167:551-61. doi:10.1001/archinte.167.6.551

7. Global initiative for Obstructive Lung Disease. Global Strategy for the Diagnosis, Management and Prevention of Chronic Obstructive Pulmonary Disease. Updated 2009. Executive Summary. Available at http://www.goldcopd.org/ Guidelineitem. asp? $|1=2 \&| 2=1$ \&intld $=2180$. Last accessed 25th January 2010.

8. Bourbeau J, Julien M, Maltais F et al. Reduction of Hospital Utilization in Patients with Chronic Obstructive Pulmonary Disease. Arch Intern Med 2003;163:585-91. http://dx.doi.org/10.1001/archinte.163.5.585

9. Taylor SJC, Candy B, Bryar RM et al. Effectiveness of innovations in nurse led chronic disease management for patients with chronic obstructive pulmonary disease: systematic review of evidence. BMJ 2005;331:485. http://dx.doi.org/ 10.1136/bmj.38512.664167.8F

10. Skwarska E, Cohen G, Skwarski KM et al. Randomized controlled trial of supported discharge in patients with exacerbations of chronic obstructive pulmonary disease. Thorax 2000;55:907-12. http://dx.doi.org/10.1136/thorax.55.11.907

11. Cotton MM, Bucknall CE, Dagg KD et al. Early discharge for patients with exacerbations of chronic obstructive pulmonary disease: a randomized controlled trial. Thorax 2000;55:902-06. http://dx.doi.org/10.1136/thorax.55.11.902

12. Ram FS, Wedzicha JA, ,Wright J et al. Hospital at home for patients with acute exacerbations of chronic obstructive pulmonary disease: systematic review of evidence. BMJ 2004;329:315. http://dx.doi.org/10.1136/bmj.38159.650347.55

13. Sridhar M, Taylor R, Dawson $\mathrm{S}$ et al. A nurse led intermediate care package in patients who have been hospitalised with an acute exacerbation of chronic obstructive pulmonary disease. Thorax 2008;63:194-200. http://dx.doi.org/ 10.1136/thx.2007.077578

14. Kunz R, Oxman AD. The unpredictability paradox: review of empirical comparisons of randomised and non-randomised clinical trials. BMJ 1998;317:1185-90.

15. Brooks D, Fancott CA, Falter LB et al. The development of a helpline for chronic obstructive pulmonary disease. Patient Education and Counselling 2004;54:32936. http://dx.doi.org/10.1016/j.pec.2003.06.001

16. Roberts MM, Leeder SR, Robinson TD. Nurse-led 24-h hotline for patients with chronic obstructive pulmonary disease reduces hospital use and is safe. Intern Med J 2008;38:334-40. http://dx.doi.org/10.1111/j.1445-5994.2007.01519.x

17. Coultas D, Frederick J, Barnett B et al. A randomized trial of two types of nurseassisted home care for patients with COPD. Chest 2005;128:2017-24. http://dx.doi.org/10.1378/chest.128.4.2017 